\title{
Brassinosteroids Suppress Rice Defense Against Root-Knot Nematodes Through Antagonism With the Jasmonate Pathway
}

\author{
Kamrun Nahar, ${ }^{1}$ Tina Kyndt,, ${ }^{1}$ Bettina Hause, ${ }^{2}$ Monica Höfte, ${ }^{3}$ and Godelieve Gheysen ${ }^{1}$ \\ ${ }^{1}$ Department of Molecular Biotechnology, Ghent University, Coupure links 653, B-9000 Ghent, Belgium; ${ }^{2}$ Institute of Plant \\ Biochemistry, Weinberg 3, D-06120 Halle/Saale, Germany; ${ }^{3}$ Department of Crop Protection, Ghent University, Coupure links \\ 653, B-9000 Ghent, Belgium
}

Submitted 8 May 2012. Accepted 3 September 2012.

\begin{abstract}
The importance of phytohormone balance is increasingly recognized as central to the outcome of plant-pathogen interactions. Next to their well-known developmental role, brassinosteroids (BR) were recently found to be involved in plant innate immunity. In this study, we examined the role of $\mathrm{BR}$ in rice (Oryza sativa) innate immunity during infection with the root-knot nematode Meloidogyne graminicola, and we studied the inter-relationship with the jasmonate (JA) pathway. Exogenous epibrassinolide (BL) supply at low concentrations induced susceptibility in the roots whereas high concentrations of BL enforced systemic defense against this nematode. Upon high exogenous BL supply on the shoot, quantitative reverse-transcription polymerase chain reaction (qRT-PCR) confirmed a strong feedback inhibitory effect, leading to reduced BR biosynthesis in the root. Moreover, we demonstrate that the immune suppressive effect of $B R$ is at least partly due to negative cross-talk with the JA pathway. Mutants in the BR biosynthesis or signaling pathway accumulate slightly higher levels of the immediate JA-precursor 12-oxo-phytodienoic acid, and qRT-PCR data showed that the BR and JA pathway are mutually antagonistic in rice roots. Collectively, these results suggest that the balance between the BR and JA pathway is an effective regulator of the outcome of the rice-M. graminicola interaction.
\end{abstract}

Plants have evolved a range of defense mechanisms in which plant hormones fulfill central roles to effectively protect themselves from microbial pathogens. The importance of the classical hormones salicylic acid (SA), ethylene (ET), and jasmonate (JA) for the plant innate immune system is well established (Grant and Jones 2009; Robert-Seilaniantz et al. 2011). Each of these hormones generates and transmits distinct defense signals and they influence each other through a complex network of synergistic and antagonistic interactions (Koornneef and Pieterse 2008; Pieterse et al. 2009), allowing the plant to efficiently tailor its defense reaction depending on the type of attacker encountered. In many cases, ET has been shown to act as an important modulator of plant responses to either SA or JA (Adie et al. 2007). In both dicots and monocots, mostly synergistic interactions between JA and ET signaling pathways have been reported, where both JA and ET are

Corresponding author: G. Gheysen; E-mail: godelieve.gheysen@ugent.be

(C) 2013 The American Phytopathological Society often required for the activation of defense-related genes, while SA and JA/ET defense pathways are found as mutually antagonistic (Beckers and Spoel 2006; Lorenzo and Solano 2005; van Loon et al. 2006).

Newly emerging critical regulators of plant-microbe interactions include other plant hormones, such as abscissic acid (ABA), brassinosteroids (BR), gibberellic acid (GA), cytokinins, and auxins. Increasing evidence suggests that these hormones render a positive or negative role in disease occurrence and interact with the SA-JA-ET signaling system (Pieterse et al. 2009; Robert-Seilaniantz et al. 2011). However, being only recently discovered, their overall mechanism of action is not completely understood.

BR compose a recently discovered group of plant hormones. These polyhydroxysteroids offer a wide range of physiological activities starting from seed germination till flowering and senescence (Azpiroz et al. 1998; Clouse and Sasse 1998; Mandava 1988; Sakurai 1999). Next to their important developmental role, BR have more recently been demonstrated to be involved in plant responses to a broad spectrum of environmental stresses that include temperature, drought, salinity, and insect herbivory (Bajguz and Hayat 2009; Belkhadir et al. 2012). It has been demonstrated that exogenous treatment with the biologically most active BR, brassinolide (BL), activates resistance in tobacco plants against Tobacco mosaic virus, the bacterial pathogen Pseudomonas syringae, and the fungal pathogen Oidium sp., and in rice plants against Magnaporthe grisea and Xanthomonas oryzae, which cause rice blast and bacterial blight, respectively (Nakashita et al. 2003). Data and results from both field and greenhouse experiments demonstrated the protective effects of exogenous BR against a broad range of fungal, viral, and bacterial pathogens, establishing a role for BR in both monocot and dicot plant defense mechanisms (Bajguz and Hajat 2009). However, certain concentrations of BR, and application of BR at certain developmental stages of the plant, can stimulate fungal growth and disease progression. For instance, BR induce susceptibility of the potato tuber tissues by stimulating the mycelial growth and intensity of spore formation of Phytophthora infestans, and weakening of the immune status of plant tissues was observed for at least four months after the treatment of whole potato tubers with BR (Vasyukova et al. 1994). Moreover, it was recently shown that exogenous application of BR also induces susceptibility to Pythium graminicola in rice roots (De Vleesschauwer et al. 2012).

Compared with the vast information on the role of BR in plant growth and development (Clouse and Sasse 1998; Fujioka 
and Yokota 2003), far less information is available on the function of this class of hormones in plant innate immunity. Molecular changes associated with BR-induced plant defense responses do not represent solely BR-linked primary responses. Recent molecular studies support the hypothesis that there is cross-talk between BR and other phytohormones (Bouqin et al. 2001; Friedrichsen et al. 2002; Goda et al. 2002; Lin et al. 2003; Müssig et al. 2000, 2002; Yi et al.1999). For instance, the interaction of BR with auxin produces a synergistic effect whereas, with GA, the effect was reported to be additive (Khripach et al. 1999; Mandava 1988). In tobacco, BR-induced resistance is not associated with an increase in SA levels or induction of pathogenesis-related (PR) gene expression (Nakashita et al. 2003). $\mathrm{BR}$ have also been reported to be antagonistic to ABA because of observed differences in gene regulation of these two hormone pathways in Arabidopsis (Abraham et al. 2003). Simultaneous treatment of tobacco and rice plants with systemic acquired resistance inducers benzothiadiazole (a SA analogue) and $\mathrm{BR}$ produced an additive protection against foliar microbes (fungi, bacteria, and virus), indicating a parallel activation of defense responses (Nakashita et al. 2003). Nevertheless, in rice roots, De Vleesschauwer and associates (2012) established that the BR pathway suppresses plant innate immunity against $P$. graminicola through its antagonistic interaction with the SA and GA pathway. Taken together, these findings strongly suggest that the mechanism by which BR induce susceptibility or resistance against pathogens is dependent on the hormone concentration and timing, and involves the activation or suppression of other hormone pathways.

In general, the interaction and overall mechanism of hormone pathways and their role in systemic and local defense mechanisms remains to be further elucidated for monocotyledonous plants where, certainly, the root immune system is largely unstudied. Aiming to further decipher the molecular logic of BRmediated defense mechanisms, we have analyzed the role of $\mathrm{BR}$ in the interaction between rice and the root-knot nematode (RKN) Meloidogyne graminicola. Rice (Oryza sativa) is unique, not only rendering a wealth of genomic and molecular information as a laboratory monocot model plant but also serving as a staple food for more than half the world's population, in contrast to other experimental model plants, such as Arabidopsis thaliana, used in plant-microbe interaction research (Jung et al. 2008).

M. graminicola has emerged as a strong candidate to be causing a consistent yield decline in aerobic rice linked to the process of so-called "soil sickness" (Bridge et al. 2005). M. graminicola is an obligate, sedentary endoparasite (Abad et al. 2003) with a wide host range (Whitehead 1998). The nematodes are called RKN because they induce the formation of hook-like galls on the roots, which gradually reduce nutrient and water uptake (Williamson 1998). The infective second-stage juveniles (J2s) of $M$. graminicola do not invade rice roots growing in flooded soils but they are able to survive in flooded soil for 5 months or more. Once inside the roots, they migrate intercellularly toward the meristem and toward the region of cell differentiation, where they initiate permanent feeding sites (Williamson and Gleason 2003). These large, multinucleated, metabolically active giant cells serve as a source of nutrients for the nematodes, which further develop into females. The females lay their eggs in mass inside the galls and juveniles hatch from the eggs and reinfect the same root. In well-drained soil at 22 to $29^{\circ} \mathrm{C}$, the life cycle of $M$. graminicola is completed in 19 days, leading to the rapid build-up of damaging population sizes within a single rice growing season (Shrestha et al. 2007). Recently, our research has shown that exogenous application of JA or ET onto rice shoots induces a systemic defense against infection by the RKN M. graminicola (Nahar et al. 2011). ET-induced systemic defense involves ET signaling and a strong activation of JA biosynthesis and signaling genes, indicating that the JA pathway is a key defense pathway involved in rice root innate immunity against RKN, modulated by ET. The SA pathway, on the other hand, has a minor positive effect on root immunity against RKN (Nahar et al. 2011). We have also shown that the RKN can actively suppress local and systemic hormone signaling pathways within the rice plant early upon infection (Kyndt et al. 2012a and b; Nahar et al. 2011).

In this work, we wanted to elucidate the role of the BR pathway in rice innate immunity against RKN. To this end, we investigated i) the nematode susceptibility of BR biosynthesis and signaling mutants, ii) the effects of different concentrations of exogenous application of BL (as a source of BR) and a $\mathrm{BR}$ biosynthesis inhibitor on rice basal defense to $M$. graminicola, and iii) the interaction between the BR pathway and the JA pathway in rice roots. We demonstrate that the BR pathway negatively impacts innate immunity in rice in a dose-dependent manner, and reveal its antagonism with the JA pathway in planta. These results unveil another dimension of the complex cross-talk among hormone-induced defense pathways in rice.

\section{RESULTS}

\section{Dose-dependent suppression of rice basal immunity by BR to the root pathogen $M$. graminicola.}

In addition to their critical roles in growth regulation, BR have recently emerged as interesting players in plant defense against biotrophic-hemibiotrophic pathogens (Nakashita et al. 2003). To determine the effect of externally supplied BR on rice defense against RKN infection in a compatible interaction, rice plants were foliarly treated with different concentrations of $\mathrm{BL}$ (as a source of BR). We studied the activation of systemic rice defense pathways and the effect on rice root susceptibility to the RKN M. graminicola. To verify the effect of foliar hormone applications on systemic defense pathways in the rice root system, the expression of BR biosynthesis and signaling genes was investigated using quantitative reverse-transcription polymerase chain reaction (qRT-PCR) in the rice roots of 'Taichung 65' (T65) $24 \mathrm{~h}$ after foliar treatment with different concentrations $(0.1,1,5$, and $10 \mu \mathrm{M})$ of exogenous BL. To this end, three genes were selected: i) OsD11, which encodes a cytochrome P450 (CYP724B1) involved in the early steps of BR biosynthesis (Tanabe et al. 2005); ii) OSDWARF, whose product catalyzes a late step in BR biosynthesis (Hong et al. 2002); and iii) the BR receptor BRASSINOSTEROID INSENSITIVE1 (OSBRII) that encodes a putative protein kinase highly similar to the Arabidopsis BRI1 protein (Yamamuro et al. 2000).

At $24 \mathrm{~h}$ after foliar treatment with a low BL concentration $(0.1 \mu \mathrm{M})$, there was a significant upregulation of both BR biosynthesis and signaling genes in rice roots in comparison with roots from the control treatment (Fig. 1A). When treated with $1 \mu \mathrm{M}$ BL, mRNA transcripts levels of OsDWARF and OsBRII were downregulated in the roots while the early biosynthesis gene $O s D 11$ still showed higher transcript levels. On the other hand, both BR biosynthesis genes were negatively regulated in rice roots after foliar treatment with high concentrations ( 5 and $10 \mu \mathrm{M})$ of BL. Feedback inhibition was also clearly seen for the signaling gene OsBRII (Fig. 1A) upon application with high levels of BL. Therefore, these results suggest that high concentrations of external BL suppress the expression of genes involved in both BR biosynthesis and signaling, regulating the internal bioactive BR levels through a negative-feedback mechanism.

Given the observed transcriptional alterations in the rice roots upon foliar external BL application, the effect of different BL concentrations on subsequent infection with $M$. grami- 
nicola was monitored. The nematodes were inoculated on the rice roots $24 \mathrm{~h}$ after $\mathrm{BL}$ treatment and, 2 weeks later, the numbers of galls per plant were counted. Results show that low concentrations of BL $(0.1$ and $1 \mu \mathrm{M})$ favored subsequent infection with $M$. graminicola as compared with the nontreated control (Fig. 1B), implying a role for BR in rice susceptibility to this nematode. However, exogenously applying high concentrations of BL ( 5 and $10 \mu \mathrm{M})$ resulted in significantly lower susceptibility toward $M$. graminicola compared with untreated plants (Fig. 1B), which can be explained by the fact that the negative feedback regulation results in lower endogenous BR levels and, hence, an increase in basal defense.

To further investigate the immune-regulatory role of BR, we next evaluated the susceptibility of rice mutants that are either deficient in or insensitive to BR. Both BR-deficient $d 2-1$ and d2-2 mutants showed significantly lower susceptibility compared with control wild-type plants (T65), whereas complementing both deficient mutants with a low $(0.1 \mu \mathrm{M})$ exogenous $\mathrm{BL}$ concentration restored the susceptibility to $M$. graminicola
(Fig. 1C). However, the scenario is again reversed when applying high concentrations $(10 \mu \mathrm{M})$ of BL (Fig. 1C), confirming the already observed feedback effect. In accordance with our observations in biosynthesis mutants, treatment with different concentrations ( 1 and $10 \mu \mathrm{M})$ of the highly specific BR biosynthesis inhibitor (Asami et al. 2000) brassinazole (BRZ) resulted in a substantial reduction in disease severity (Fig. 1C; data not shown for $10 \mu \mathrm{M})$. Mutant $d 61-1$ that carries a lossof-function mutation in the rice BR receptor gene OsBRII also showed a lower susceptibility compared with wild-type plants but, in line with its insensitivity (Yamamuro et al. 2000), exogenous BL treatment did not have an effect on the susceptibility of d61-1 toward RKN (Fig. 1D). Collectively, these data indicate that both internal BR levels and subsequent BR signaling promote susceptibility of rice roots to $M$. graminicola.

\section{$B R$ and $J A$ are antagonistic in rice roots.}

Mounting evidence indicates that defense signaling is not a linear single response event but, instead, involves a complex
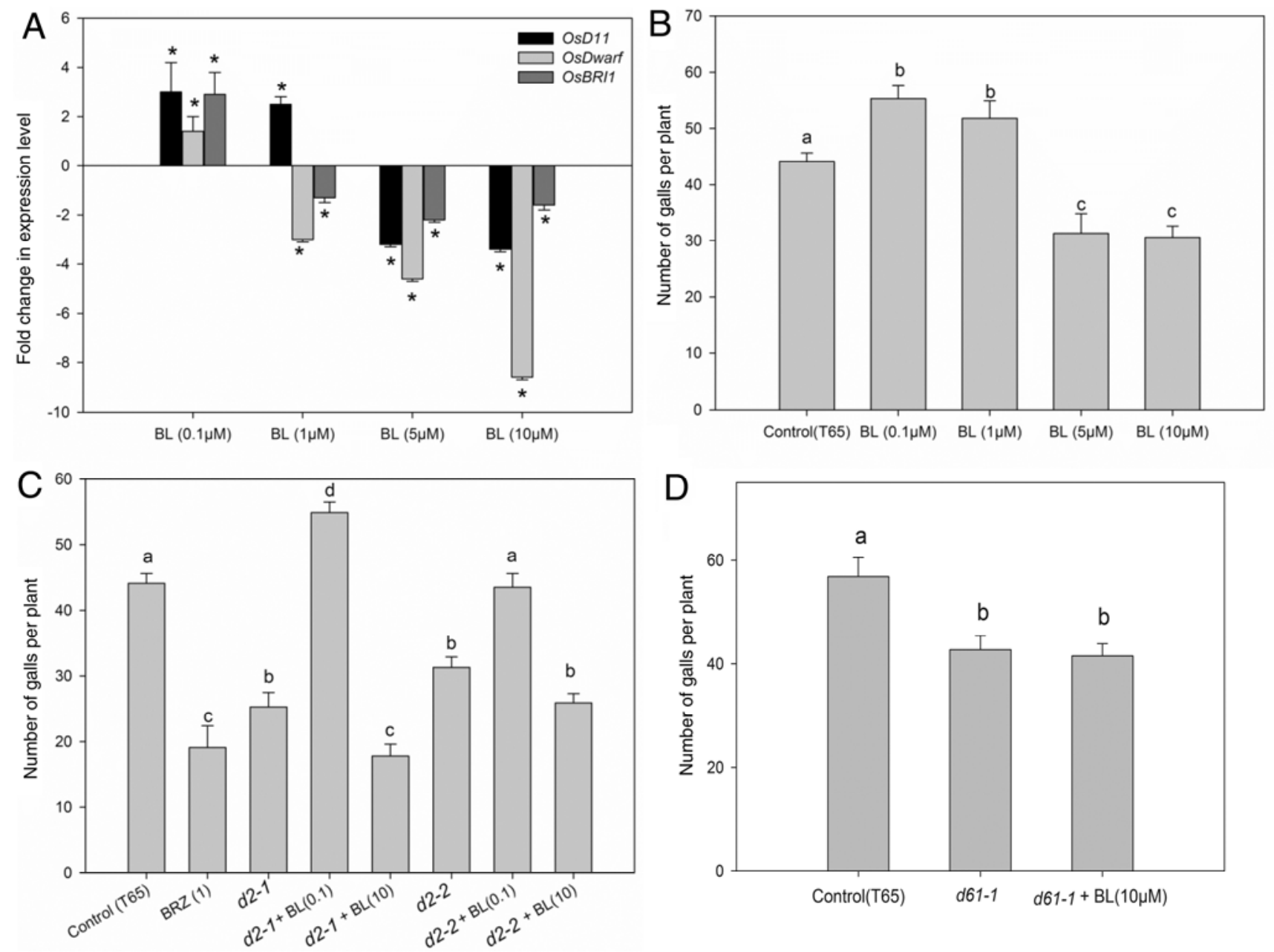

Fig. 1. Low levels of exogenous brassinosteroids (BR) suppress root immunity in rice against the root-knot nematode Meloidogyne graminicola. A, Quantitative reverse-transcription polymerase chain reaction (qRT-PCR) data showing the response of the BR pathways in rice roots at $24 \mathrm{~h}$ after epibrassinolide (BL) treatment at different concentrations. qRT-PCR was executed on RNA from root tissue $24 \mathrm{~h}$ after treatment. The relative expression level (fold change) of the selected genes in root tissues of treated versus untreated plants is shown. Gene expression levels were normalized using two internal reference genes, OsEXP and OsEXPNarsai. Bars represent the mean and standard error of two biological replicates, each containing a pool of three plants. Asterisks indicate statistically significant $(P<0.01)$ differential expression in comparison with untreated plants. B, Effect of BR-induced response on wild-type 'Taichung 65' (T65) plants against $M$. graminicola with different concentrations of BL foliar treatment. Fifteen-day-old T65 plant shoots were sprayed until runoff with different concentrations of BL $(0.1,1,5$, and $10 \mu \mathrm{M})$ or the corresponding control solution. $\mathbf{C}$ and $\mathbf{D}$, Disease-reducing effect of exogenously applied brassinazole (BRZ), a BR biosynthesis inhibitor, and effect of exogenous treatment of BL $(0.1$ and $10 \mu \mathrm{M})$ in BR-deficient $(d 2-1$ and $d 2-2)$ mutants and the insensitive mutant (d61-1) against $M$. graminicola. Plant roots were inoculated with 300 second-stage juveniles of $M$. graminicola. Bars represent the mean and standard error of the number of galls 2 weeks after inoculation recorded on eight plants. For infection experiments, different letters indicate statistically significant differences (Duncan multiple range test with $\alpha=0.05$ ). 
network between different signals and effectors (Koornneef and Pieterse 2008). Therefore, to further decipher the molecular machinery underpinning BR-modulated rice defense to nematodes, the interaction of BR with other pathways was explored. Given the eminent importance of the JA pathway in systemically induced defense against RKN in rice (Nahar et al. 2011) and the recent findings of BR cross-talk with other signal compounds in the context of abiotic and biotic stress signaling (Bajguz and Hayat 2009; De Vleesschauwer et al. 2012; Divi et al. 2010), we analyzed the interaction between the BR and JA pathway.

In addition to the differential expression of BR biosynthesis and signaling genes in rice roots treated with different external
BL concentrations (Fig. 1A), transcriptional alterations were also detected in two tested genes from the JA pathway: biosynthesis gene OsAOS2 (Mei et al. 2006) and JA-induced signaling gene OsJAmyb (Lee et al. 2001) (Fig. 2A). Both JA-related gene transcripts were strongly downregulated in BL-treated rice roots at $24 \mathrm{~h}$ after foliar hormone treatment (Fig. 2A), when the BL concentration was low $(0.1$ and $1 \mu \mathrm{M})$. In contrast, the JA genes were upregulated when the plants were treated with high concentrations $(5$ and $10 \mu \mathrm{M})$ of BL. These data suggest a negative interaction between the BR and JA pathway in planta. In support of this assumption, exogenous foliar spraying with methyl jasmonate (MeJA) $(100 \mu \mathrm{M})$ resulted in strong downregulation of the BR biosynthesis and
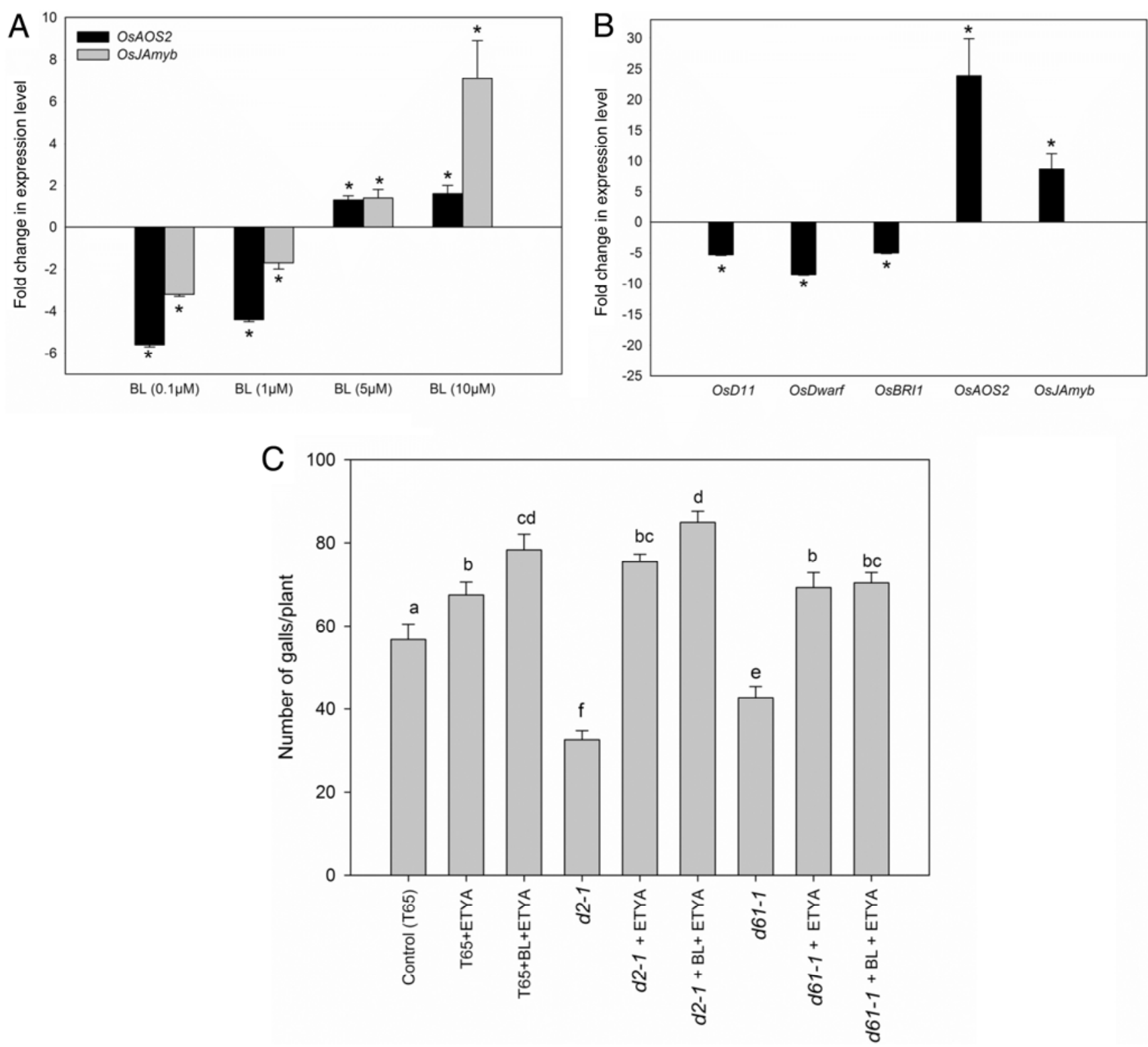

Fig. 2. Brassinosteroids (BR) are antagonistic to the jasmonate (JA) pathway in rice roots. A, Quantitative reverse-transcription polymerase chain reaction (qRT-PCR) data showing the response of the JA pathways in rice roots at $24 \mathrm{~h}$ after treatment with different concentrations of epibrassinolide (BL), B, Crosstalk analysis showing the negative interaction between BR and JA in root tissue of 'Taichung 65' (T65) treated with methyl jasmonate (100 $\mu$ M). qRT-PCR was executed on RNA from root tissue $24 \mathrm{~h}$ after treatment. The relative expression level (fold change) of the selected genes in root tissues of treated versus untreated plants is shown. Gene expression levels were normalized using two internal reference genes, OsEXP and OsEXPNarsai. Bars represent the mean and standard error of two biological replicates, each containing a pool of three plants. Asterisks indicate statistically significant $(P=0.001)$ differential expression in comparison with untreated plants, $\mathbf{C}$, Number of galls/plant after treatment of the wild type and BR-deficient and insensitive mutants with JA biosynthesis-inhibitor 5,8,11,14-eicosatetraynoic acid (ETYA) $(100 \mu \mathrm{M})$ alone or in combination with BL $(0.1 \mu \mathrm{M})$. Fifteen-day-old plants were sprayed until run-off with the inhibitor, the combination with BL, or the control solution. Plant roots were inoculated with 300 second-stage juveniles of Meloidogyne graminicola. Bars represent the mean and standard error of the number of galls 2 weeks after inoculation recorded on eight plants. For infection experiments, different letters indicate statistically significant differences (Duncan multiple range test with $\alpha=0.05$ ). 
signaling genes in the rice roots (Fig. 2B) (OSDWARF, 8.5fold; $O s D 11,5.3$-fold; and OsBRI1, 5.0-fold), indicating that BR and JA interact in a mutually antagonistic manner in the rice root. Given the strong negative feedback regulation of $\mathrm{BR}$ biosynthesis when externally supplying high concentrations of $\mathrm{BL}$ to the rice shoot (Fig. 1A), we hypothesized that the decrease in disease susceptibility observed in plants treated with 5 to $10 \mu \mathrm{M}$ BL (Fig. 1B) resulted from reduced levels of endogenous BR and from elevated levels of endogenous JA, where JA induces defense (Nahar et al. 2011).

To further examine the nature of the BR-JA interaction, an infection experiment was conducted where JA biosynthesis was inhibited through the lipoxygenase (LOX) inhibitor 5,8,11,14-eicosatetraynoic acid (ETYA). Blocking JA biosynthesis in the wild type and in the BR biosynthesis mutants significantly increased the plants' susceptibility toward RKN (Fig. 2C). Furthermore, co-application of BL $(0.1 \mu \mathrm{M})$ with ETYA resulted in $20 \%$ more susceptibility relative to treatment with either compound alone (Fig. 2C). However, BR accumulation did not appear to be a prerequisite for ETYA-induced susceptibility, because ETYA treatment was equally effective in the wild type and BR-deficient (d2-1) and insensitive (d61-1) mutants. Moreover, co-treatment of ETYA with BL $(0.1 \mu \mathrm{M})$ in a BR-deficient $d 2-1$ plant caused an additional and significant increase $(35 \%)$ in disease susceptibility compared with treatment with either compound alone (ETYA or BL) (Fig. 2C). This suggests that BR can still induce increased susceptibility toward $M$. graminicola when the endogenous JA content in rice roots is low, indicating effects of BR in addition to antagonism of the JA pathway. In light of the results presented in Figure 2, we suggest that the BR pathway induces susceptibility in rice toward $M$. graminicola, at least partly through its antagonism with the JA pathway in the roots.

Finally, to further validate the antagonistic interaction of BR with the rice JA pathway, the endogenous levels of different oxylipins-i) JA, ii) the biologically active jasmonic acid derivative $\mathrm{JA}$-isoleucine (JA-Ile), and iii) the immediate JA precursor 12-oxo-phytodienoic acid (OPDA) - were measured in roots of wild-type and BR mutant rice plants. Results show that the endogenous levels of all three JA were, on average, higher in the $d 61-1 \mathrm{BR}$ signaling mutant compared with the wild-type T65, although the data were not statistically significant. On the other hand, the $d 2-2$ biosynthesis mutant accumulated significantly higher levels of OPDA (Table 1), an active oxylipin involved in defense in the absence of JA (Rakwal et al. 2002; Stelmach et al. 1998; Stintzi et al. 2001). Although the $d 61-1$ mutant accumulated endogenous OPDA at a level which was even higher than the $d 2-2$ mutant, the difference was not significant because of very high standard deviations within the $d 61-1$ data set. These results indicate that the lower susceptibility of BR biosynthesis and signaling mutants (Fig. 1C) could at least partly be due to increased endogenous OPDA levels, in combination with lower BR levels (Table 1) (Hong et al. 2002).
BR- and JA-regulated gene expression on wild-type and BR mutants in response to exogenous $B L$.

In order to obtain a more detailed understanding of the BRJA cross-talk, qRT-PCR was used to monitor the mRNA levels of BR- and JA-related genes in roots of the wild type and BR biosynthesis mutants in the absence or presence of different concentrations of BL. The relative expression levels of BR and JA pathway genes in roots of the wild type and BR mutants with or without foliar application with low $(0.1 \mu \mathrm{M})$ or high $(10 \mu \mathrm{M})$ concentrations of $\mathrm{BL}$ are shown in Figure $3 \mathrm{~A}$ and $\mathrm{B}$, respectively. Both BR-deficient $d 2$ mutants showed a significantly enhanced transcript accumulation of JA biosynthesis (OsAOS2) and signaling (OsJAmyb) genes compared with wild-type T65, confirming JA-BR antagonism in rice roots. Both mutants do not show differential expression of the signaling gene OsBRII compared with control T65. On the other hand, when the mutants were foliarly treated with low concentrations of BL $(0.1 \mu \mathrm{M})$, an activation of $O s D 11$ was observed in the roots, whereas the transcript levels of OSDWARF and OsBRI1 were downregulated. In comparison, transcript levels of JA-related genes $(O s A O S 2$ and $O s J A m y b)$ were downregulated in both $d 2$ mutants after application of low BL concentrations (Fig. 3A), corroborating that BR treatment $(0.1 \mu \mathrm{M})$ suppresses the JA pathway.

For high concentrations of BL foliar treatment, both the wild type and BR mutants show that, when the BR biosynthesis is strongly downregulated, mRNA levels of JA biosynthesis and signaling genes are elevated (Fig. 3B). In similar vein with our previous observations of feedback inhibition, BR biosynthesis and signaling genes were strongly negatively regulated or had returned to basal expression level upon exogenous treatment with high concentrations of BL $(10 \mu \mathrm{M})$ in both wild-type T65 and BR mutant plants (Fig. 3B).

In general, at either low or high concentrations of $\mathrm{BL}$ and in the wild type and BR mutants, an up- or downregulation of BR biosynthesis always counteracts with a down- or upregulation of the JA pathway, respectively. These results confirm that JA and BR mutually antagonize each other's signaling pathway in rice roots. When considered together, our results support a model (Fig. 4) whereby endogenous BR levels suppress rice root innate defense against $M$. graminicola attack, at least in part by antagonizing the JA pathway.

\section{DISCUSSION}

The balance of hormonal cross-talk strongly influences the outcome of plant-pathogen interactions by modulating plant defense pathways in various ways (Robert-Seilaniantz et al. 2011). In this study, we focused on one of the less-studied plant hormones, BR. It has been established that BL, castasterone (CS), and their related compounds are biosynthesized from campesterol (Fujioka et al. 1997; Sakurai and Fujioka 1997; Yokota et al. 1997) and BL is the most biologically active BR. BR play important roles in physiological and develop-

Table 1. Levels of free 12-oxo-phytodienoic acid (OPDA), jasmonate (JA), and JA-isoleucine (JA-Ile) (mean \pm standard deviation), and brassinosteroid (BR) (Castasterone level) (Hong et al. 2002) in wild-type Oryza sativa 'Taichung 65' (T65) and corresponding BR mutants ${ }^{\mathrm{y}}$

\begin{tabular}{lccc}
\hline Rice line & OPDA $(\mathbf{p m o l} / \mathbf{g ~ F W})$ & JA $(\mathbf{p m o l} / \mathbf{g}$ FW) & JA-Ile $(\mathbf{p m o l} / \mathbf{g}$ FW) \\
\hline T65 & $174.0 \pm 59.0 \mathrm{a}$ & $79.7 \pm 62.9 \mathrm{a}$ & $114.8 \pm 14.8 \mathrm{a}$ \\
$d 2-2$ & $366.4 \pm 101.0 \mathrm{~b}$ & $57.6 \pm 26.7 \mathrm{a}$ & $107.7 \pm 33.7 \mathrm{a}$ \\
$d 61-1$ & $407.6 \pm 348.3 \mathrm{a}$ & $99.2 \pm 42.4 \mathrm{a}$ & $126.7 \pm 23.0 \mathrm{a}$ \\
\hline
\end{tabular}

${ }^{\mathrm{y}}$ Rice seedlings were grown for 15 days, roots were sampled from wild-type T65 and BR biosynthesis (d2-2) and signaling (d61-1) mutants, and the JA content was determined by gas chromatography-mass spectrometry analysis. Results are the mean from five biological replicates each containing a pool of three plants. FW = fresh weight; $*=$ endogenous BR level in $d 61-2$ mutant (Yamamuro et al. 2000), $d 61-1$ and $d 61-2$ are allelic mutations in the same gene. Within a column, means having the same letter do not differ significantly while means with different letters are significantly different $(P<0.01)$.

${ }^{\mathrm{z}}$ Data from Hong et al. 2002 and Yamamuro et al. 2000. 
mental processes, including seed germination, skotomorphogenesis, flowering, and senescence (Clouse and Sasse 1998). Next to their role as plant growth regulators, an impact of BR on plant defense has also been demonstrated (Belkhadir et al. 2012) but the defense regulatory roles of BR and their crosstalk with other hormones in rice and, more specifically, in rice-pathogen interactions is largely unstudied. However, recently, De Vleesschauwer and associates (2012) have shown that the BR pathway is involved in rice root susceptibility to the root pathogen $P$. graminicola. Interestingly, this pathogen seems to hijack the BR pathway to suppress the SA- and GAmediated signal transduction pathways that are involved in disease resistance (De Vleesschauwer et al. 2012). The research described here aims to provide a further characterization of the role of BR-mediated defense signaling in rice and its importance in rice root interactions with the RKN M. graminicola. By using BR biosynthesis and signaling mutants and exogenous applications in this model monocot plant system, a physiologically important function could be demonstrated for BR in susceptibility of rice against this root pathogen. Measurements of JA and OPDA levels in BR mutants and infection analyses using BR mutants revealed that the BR-mediated susceptibility works at least partly through its antagonism with the JA pathway in the rice roots. This was further elaborated by qRT-PCR analyses of genes involved in these pathways and chemical blocking of JA and BR biosynthesis, with the results pointing to a mutual antagonistic interaction between $\mathrm{BR}$ and JA in rice roots.

\section{Dose-dependent effect of BR}

through a negative-feedback mechanism in the rice root.

Exogenous treatment with high $(40$ to $200 \mu \mathrm{M})$ concentrations of BL has previously been shown to activate resistance in tobacco plants against Tobacco mosaic virus, the bacterial pathogen Pseudomonas syringae pv. tabaci, and the fungal pathogen Oidium sp., and in rice plants against rice blast and bacterial blight (Nakashita et al. 2003). However, recently, it has been demonstrated that the BR homeostasis in the plant controls the plant innate immunity in Arabidopsis (Belkhadir et al. 2012). Thus, the concentration and timing of BR applica-
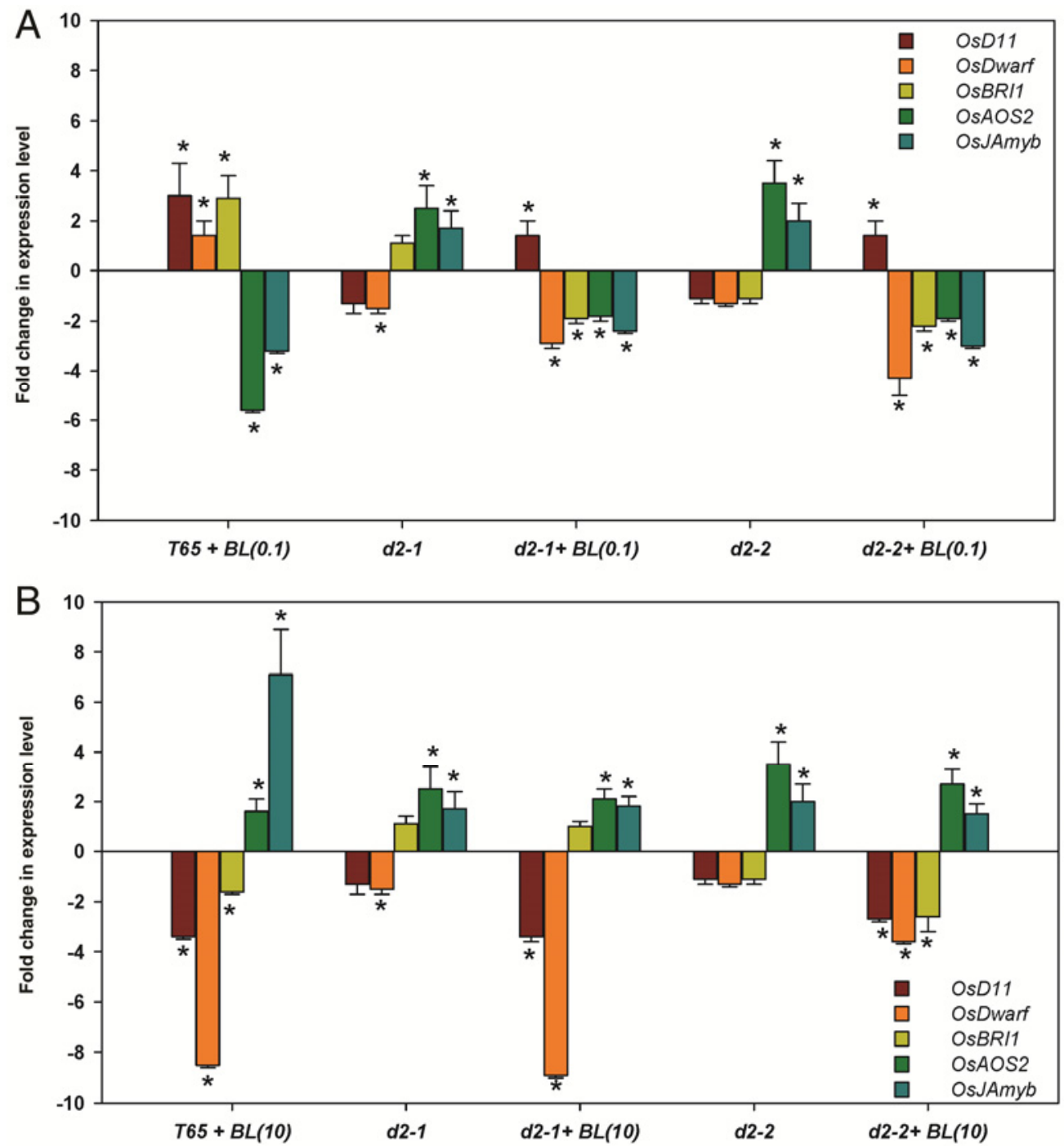

Fig. 3. Quantitative polymerase chain reaction-(qPCR) data on some selected brassinosteroid (BR) and jasmonate (JA) pathway genes in wild-type 'Taichung 65' (T65) and BR mutant rice roots. Effects of $\mathbf{A}$, low $(0.1 \mu \mathrm{M})$ and $\mathbf{B}$, high $(10 \mu \mathrm{M})$ concentrations of epibrassinolide (BL) on these plants on the expression of these genes is shown. Reverse-transcription qPCR was executed on RNA from root tissue of 15-day-old plants and 24 h after treatment. Data are shown as relative expression levels of untreated (mutant) and treated (wild-type and mutant) tissue in comparison with the control tissue (root tissue from untreated T65 plants. Gene expression levels were normalized using two internal reference genes, OsEXP and OsEXPNarsai. Bars represent mean expression levels and standard error from two biological replicates, each containing a pool of three plants. Asterisks indicate statistically significant $(P<0.01)$ differential expression in comparison with untreated plants. 
tion seem to be important factors to be considered in BR-related research. Indeed, our data show a remarkable discrepancy between high and low concentrations of BL treatments, with high concentrations (5 and $10 \mu \mathrm{M})$ resulting in plant resistance against RKN while low concentrations $(0.1$ and $1 \mu \mathrm{M})$ promote plant susceptibility. qRT-PCR data demonstrated that this phenomenon is related to negative feedback of the BR pathway in planta upon high exogenous BL supply. It has been described before that BR are present and active in extremely low concentrations in planta (Khripach et al. 2000). Previously, it was reported that BR biosynthesis and signaling genes are locally downregulated by high exogenous BL concentrations (Hong et al. 2002, 2003; Tanabe et al. 2005) but the systemically altered expression of BR biosynthesis and signaling genes in root tissues upon foliar BR supply has not been demonstrated before. Similar to the $O s D 11$ results obtained in this work in rice roots, expression of the Arabidopsis cytochrome P450 genes, DWF4/CYP90B1, CPD/CYP90A1, DWARF/ CYP85A1, ROT3/CYP90Cl, and CYP90D1 was shown to be suppressed by exogenous BL supply (Bancos et al. 2002; Goda et al. 2002). He and associates (2005) demonstrated that BR cellular homeostasis in dicotyledonous plants is achieved mainly by end-product feedback regulation with activation of the transcriptional repressor (BZR1), which binds directly to the promoters of feedback-regulated BR biosynthetic genes. These observations indicate that the negative feedback regulation of genes related to BR biosynthesis by high BL is a common phenomenon in both dicot and monocot plants. Therefore, such a mechanism of feedback regulation is an integral part of BR action, and a detailed knowledge of this mechanism at the molecular level is essential for an integrated understanding of how plants use BR to regulate development and defense.

Recent studies showed that, in pea (Pisum sativum), Arabidopsis, and tomato (Solanum lycopersicum), the levels of the

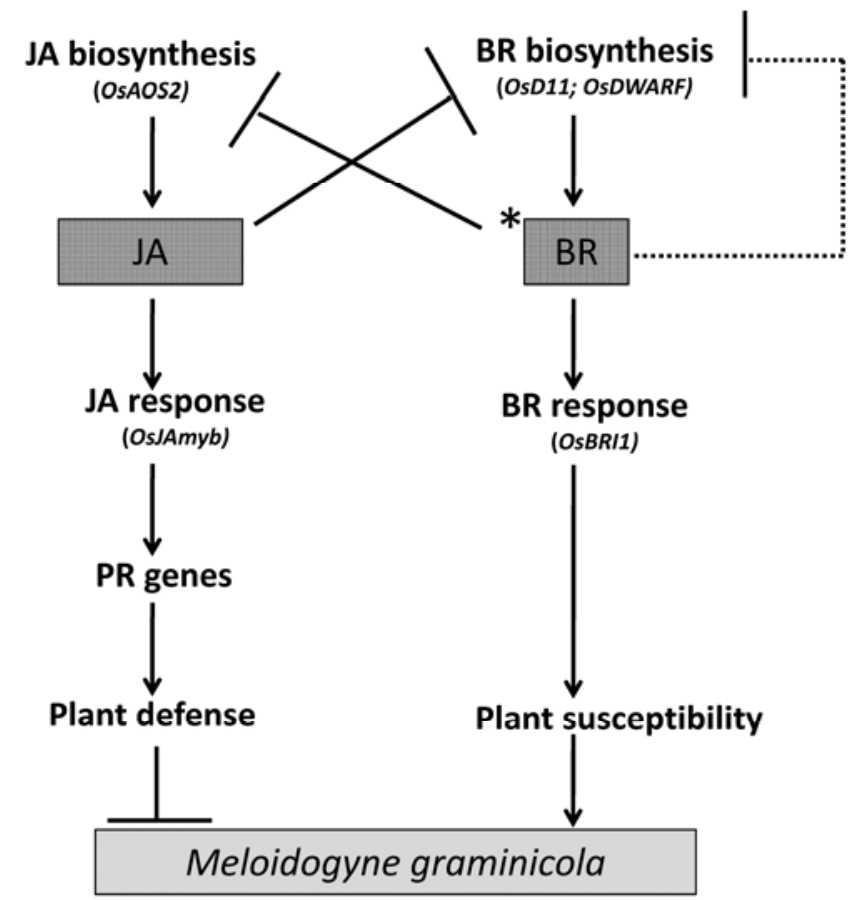

Fig. 4. Model illustrating how brassinosteroid (BR) and jasmonate (JA) interplay in induced defense in wild type rice plants. Genes shown to be involved in this mechanism are indicated in parentheses. Lines ending with arrows show activation. The solid line ending with a perpendicular short line indicates suppression or antagonistic interactions. Blunted, long dotted lines depict negative feedback regulation; * Note: excessive BR levels result in i) feedback inhibition of BR biosynthesis and ii) higher JA biosynthesis. early intermediates in the BR biosynthesis pathway were higher in the roots, while the final products of the pathway, CS and 6-DeoxoCS, were more abundant in the shoots of all three species (Symons and Reid 2004), suggesting that the roots actively participate in BR biosynthesis. Although there are no experimental data available from root tissue, we hypothesize that the rice root also contains high levels of these intermediates. Our results show that, with an application of $1 \mu \mathrm{M} \mathrm{BL}$, the late biosynthesis gene $O S D W A R F$ and the receptor gene OsBRI1 were downregulated, whereas the early biosynthesis gene $O s D 11$ was upregulated. Our observations are in accordance with the view that the maintenance of optimal local BR concentrations by coordinated biosynthetic and inactivation mechanisms, in combination with the differential responsiveness of target cells and tissues, enables the proper regulation of phytohormone physiological functions during plant development (Bancos et al. 2002) as well as plant immune responses (Belkhadir et al. 2012).

\section{Physiological BR levels suppress rice innate immunity against $R K N$, at least partly \\ through antagonism with the JA pathway.}

Experiments with low concentrations of BR show that an activated BR pathway in the rice root system results in enhanced susceptibility to the RKN. In corroboration with this view, BRbiosynthesis mutants and chemical blocking of this pathway hampers gall development on the roots. Our data suggest a model (Fig. 4) in which the existence of a narrow range of BR concentrations sensitizes the plant immune system. Recently, De Vleesschauwer and associates (2012) showed that BR antagonize with the SA pathway in rice roots to promote susceptibility for Pythium infection. SA, JA, and ET are considered to be the key players in plant innate immunity but the JA pathway has been previously demonstrated to be the most eminent pathway for rice defense against RKN (Nahar et al. 2011). That is why we decided to further decipher the interaction between the BR pathway and JA. Various types of cross-talk among phytohormone-mediated signaling in stress responses have been reported. In Arabidopsis, BR $(1 \mu \mathrm{M})$ induces the expression of OPDA reductase3, which is a JA biosynthetic enzyme (Müssig et al. 2000), and 1-aminocyclopropane-1-carboxylate synthase, which is an ET biosynthetic enzyme (Yi et al. 1999). However, whether JA or ET is required for BL-induced susceptibility in rice was unknown thus far. Our results indicate that physiologically enhanced BR levels suppress the JA pathway and vice versa in rice roots. Whether these different observations are due to the fact that dicots differ from monocots or whether they depend on the plant tissue (shoots versus roots) remains to be further investigated. In this study, we have shown that BR and JA mutually antagonize each other's pathways in rice. Application of low levels of BL suppress the expression of OsAOS2 and OsJAmyb, two important genes of the JA pathway in rice roots, and this is accompanied by an enhanced susceptibility to $M$. graminicola infection. Conversely, external JA application suppresses BRrelated gene expression. We also have indications that the endogenous levels of BR and the JA-intermediate OPDA are inversely correlated in rice roots of the wild type and BR-biosynthesis mutants. Previously, OPDA was suggested to be a signaling compound in the plant defense system without the necessity for conversion to JA (Stintzi et al. 2001). OPDA enhances resistance to both biotrophic and necrotrophic pathogens in the absence of JA in Arabidopsis (Stintzi et al. 2001). Taken together, our data strongly suggest that the balance of interaction between $\mathrm{BR}$ and JA is an important determinant for the outcome of the rice- $M$. graminicola interaction. Nevertheless, if JA biosynthesis is inhibited by ETYA application, BR still have an enhancing effect on root susceptibility, independent 
from JA. This observation was also proven by qRT-PCR-data. This may suggest two possibilities: i) BL is a better inhibitor of JA-biosynthesis than ETYA and, together with JA inhibition, results in more susceptibility relative to treatment with either compound alone; or ii) BL may act additionally through other defense pathways. Recently, De Vleesschauwer and associates (2012) have shown that both exogenously administered and endogenous BR negatively influence root immunity to the rice pathogen Pythium graminicola. Moreover, their results suggested that this immune-suppressive effect of BR is due, at least in part, to negative cross-talk with SA and GA pathways. In combination with the here-provided results, BR seem to be important immune-suppressive modulators of different defense pathways. Interestingly, our recent mRNA-Seq study (Kyndt et al. 2012a) revealed a general induction of the BR pathway in RKN-induced galls. Shan and associates (2008) reported that bacterial effectors AVRPto and AVRPtoB target the BR coreceptor $\mathrm{BAK} 1$ to modify $\mathrm{BR}$ signaling and downgrade host defenses. It remains to be further investigated whether RKN use similar BR-targeted effector mechanisms to suppress the rice immune system during their infection process.

\section{MATERIALS AND METHODS}

\section{Plant material and growth conditions.}

Rice (O. sativa) lines used in this work included the japonica T65 and the corresponding BR-insensitive d61-1 (Yamamuro et al. 2000) and BR-deficient mutant lines $d 2-1$ and $d 2-2$ (Hong et al. 2003). Seed were germinated on wet filter paper for 5 days at $30^{\circ} \mathrm{C}$, transferred to Synthetic Absorbent Polymer substrate (Reversat et al. 1999), and further grown at $26^{\circ} \mathrm{C}$ under a $12-\mathrm{h}$ light regime and 70 to $75 \%$ relative humidity. Plants were watered with distilled water once per week and fertilized twice per week with $10 \mathrm{ml}$ of Hoagland solution.

\section{Infection experiments.}

The M. graminicola culture was provided by D. De Waele (Catholic University Leuven) and was originally isolated in the Philippines. A pure nematode culture was maintained in vivo on wild-type Nipponbare plants and grasses (Echinocloa crusgalli). Nematodes were extracted from 3-month-old infected plants using the modified Baermann method (Luc et al. 2005). The nematode suspension was collected after $48 \mathrm{~h}$. Sixteen-day-old rice plants were inoculated with $300 \mathrm{~J} 2$ of $M$. graminicola per plant or mock inoculated with water. The infection level of the plants was evaluated at 15 day postinoculation using acid fuchsin staining. To visualize the galls, roots were boiled for $3 \mathrm{~min}$ in $0.8 \%$ acetic acid and $0.013 \%$ acid fuchsin. They were washed with running tap water and then destained in acid glycerol. Nematode susceptibility of the plants was evaluated by counting the number of galls per plant.

\section{Chemical treatments.}

BL and LOX inhibitor ETYA were purchased from SigmaAldrich (Bornem, Belgium) and BRZ was purchased at Wako
(Osaka Japan). BL and ETYA were dissolved in ethanol, whereas BRZ was dissolved in dimethyl sulfoxide containing $0.02 \%$ ( vol $/ \mathrm{vol}$ ) Tween 20 . The following concentrations were used: $0.1,1,5$, and $10 \mu \mathrm{M} \mathrm{BL} ; 1$ and $10 \mu \mathrm{M}$ BRZ; $100 \mu \mathrm{M}$ MeJA; and $100 \mu \mathrm{M}$ ETYA. For chemical treatment of plants, intact 15-day-old seedlings were sprayed with vaporizers until run-off with a fine mist of either compound at the indicated concentrations. Distilled water containing a few drops of ethanol and $0.02 \%(\mathrm{vol} / \mathrm{vol})$ Tween 20 was used as a control treatment. All experiments were independently repeated with similar results. In infection experiments, the chemicals were sprayed $24 \mathrm{~h}$ before nematode inoculation.

\section{Data collection and statistical analyses.}

All statistical analyses were performed in SPSS. Normality of the data was checked by applying the Kolmogorov-Smirnov test of composite normality $(\alpha=0.05)$. Homoscedasticity of the data was checked by applying the Levene test $(\alpha=0.05)$. The assumptions of normality and homoscedasticity of the data were found to be fulfilled in all cases. Collected data were then analyzed using analysis of variance. The means of the control and treated groups were compared by Duncan's multiple mean comparison test.

\section{RNA extraction, cDNA synthesis, and qRT-PCR.}

Root RNA was extracted using TRIzol (Invitrogen, Carlsbad, CA, U.S.A.) following the manufacturer's instructions. The RNA concentration and purity were measured using the NanoVue spectrophotometer (GE Healthcare, Diegem, Belgium). To remove contaminating DNA, the extract was treated with DNaseI. RNA $(1 \mu \mathrm{g})$ was treated with $1 \mu \mathrm{l}$ of DNaseI $\left(1 \mathrm{U} \mu \mathrm{l}^{-1}\right.$; Fermentas GmbH, St. Leon-Rot, Germany), $1 \mathrm{ml}$ of RiboLock RNase Inhibitor (40 units $\mu \mathrm{l}^{-1}$; Fermentas), and $1.8 \mu \mathrm{l}$ of DNaseI buffer $(10 \times$, Fermentas) in a total volume of $18 \mu \mathrm{l}$. The mixture was incubated at $37^{\circ} \mathrm{C}$ for $30 \mathrm{~min}$, after which $2 \mu \mathrm{l}$ of $25 \mathrm{mM}$ EDTA was added and incubated for $10 \mathrm{~min}$ at $65^{\circ} \mathrm{C}$ to stop the reaction. First-strand cDNA synthesis was done in three steps: i) addition of $1 \mu \mathrm{l}$ of oligo(dT) $\left(700 \mathrm{ng} \mu \mathrm{l}^{-1}\right), 2 \mu \mathrm{l}$ of $10 \mathrm{mM}$ deoxyribonucleotide triphosphates, and $4 \mu \mathrm{l}$ of RNasefree water to the DNase-treated RNA and incubation for $5 \mathrm{~min}$ at $65^{\circ} \mathrm{C}$ (to remove secondary structures); ii) addition of $8 \mu \mathrm{l}$ of $5 \times$ first-strand buffer (Invitrogen) and $4 \mu \mathrm{l}$ of $0.1 \mathrm{M}$ dithiothreitol and incubation for $2 \mathrm{~min}$ at $42^{\circ} \mathrm{C}$; and iii) addition of $1 \mu \mathrm{l}$ of SuperScript II Reverse Transcriptase (200 units $\mathrm{ml}^{-1}$; Invitrogen) and incubation for $2 \mathrm{~h}$ at $42^{\circ} \mathrm{C}$. The solution was diluted by adding $60 \mu \mathrm{l}$ of water and the quality of the cDNA was tested by performing a standard RT-PCR with some reference genes (Table 2 ) and checking the products on a $1.5 \%$ agarose gel.

The SensiMix SYBR No-ROX Kit (Bioline, London) was used in all qRT-PCR analyses. The reaction mixture contained the following ingredients: $1 \mu \mathrm{l}$ of first-strand cDNA; $10 \mu \mathrm{l}$ of $2 \times$ mastermix containing SYBR Green I dye, dNTPs, stabilizers, and enhancers; $4 \mu \mathrm{l}$ of sterilized deionized water; and $600 \mathrm{nM}$ of each primer (Table 2) in a total volume of $20 \mu \mathrm{l}$. All

Table 2. Overview of the reference and target genes used in this study, showing their GenBank accession or locus numbers and the primer pair used for quantitative reverse-transcription polymerase chain reaction

\begin{tabular}{|c|c|c|c|}
\hline Reference, or target gene & GenBank accession or locus number & Forward primer $\left(5^{\prime}-3^{\prime}\right)$ & Reverse Primer $\left(5^{\prime}-3^{\prime}\right)$ \\
\hline OsEXP & LOC_Os03g27010 & TGTGAGCAGCTTCTCGTTTG & TGTTGTTGCCTGTGAGATCG \\
\hline OsEXPNarsai & LOC_Os07g02340.1 & CACGTTACGGTGACACCTTTT & GACGCTCTCCTTCTTCCTCAG \\
\hline OsD11 & $\mathrm{AB} 1 \overline{5} 8759$ & CCACAGGAAGGCTCTCAAAG & GCTGAAATTGTTGGGCATTT \\
\hline OsDwarf & AB084385 & ATGGCCTTGCTATCTGCACT & GGGTGTAGAGCTCTGCCTTG \\
\hline OsBRII & NM_191780.1 & CTTTCTCGGCACTTTCCTTG & AGGGAACCAAGCTCTGGAAT \\
\hline OsAOS2 & NM_001055971.1 & TGCGCGACCGCCTCGATTTC & GGCCAGGCGGGACGTTGATG \\
\hline OsJAmyb & AY026332 & GAGGACCAGAGTGCAAAAGC & CATGGCATCCTTGAACCTCT \\
\hline
\end{tabular}


PCR analyses were performed in three technical replicates. Two independent biological replicates, each containing a pool of three plants, were analyzed. The reactions were performed in the Rotor-Gene 3000 (Corbett Life Science, Venlo, The Netherlands) using Rotor Discs (Qiagen, Venlo, The Netherlands), and results were generated by the Rotor-Gene 6 software. PCR was performed under the following conditions: 10 min at $95^{\circ} \mathrm{C}$ and 45 cycles of $25 \mathrm{~s}$ at $95^{\circ} \mathrm{C}, 45 \mathrm{~s}$ at $58^{\circ} \mathrm{C}$, and $25 \mathrm{~s}$ at $72^{\circ} \mathrm{C}$. After the PCR, a melting curve was generated by gradually increasing the temperature to $95^{\circ} \mathrm{C}$ to test the amplicon specificity. Data were analyzed using the REST 384 software (Corbett Research) (Pfaffl et al. 2002). This software uses a permutation analysis to compare the relative expression between a sample and a control group and to determine the statistical significance of the results.

\section{Quantitative analyses of OPDA, JA, and JA-Ile.}

Roots were harvested from the different lines, frozen in liquid nitrogen, and stored at $-80^{\circ} \mathrm{C}$ until use. Approximately 300 $\mathrm{mg}$ fresh weight of each sample was used for analysis. The amounts of OPDA, JA, and JA-Ile were determined as described by Hause and associates (2000). Statistical analyses were performed as described above.

\section{LITERATURE CITED}

Abad, P., Favery, B., Rosso, M. N., and Castagnone-Sereno, P. 2003. Rootknot nematode parasitism and host response: Molecular basis of a sophisticated interaction. Mol. Plant Pathol. 4:217-224.

Abraham, E., Rigo, G., Szekely, G., Nagy, R., Koncz, C., and Szabados, L. 2003. Light-dependent induction of proline biosynthesis by abscisic acid and salt stress is inhibited by brassinosteroid in Arabidopsis. Plant. Mol. Biol. 51:363-372.

Adie, B., Chico, J. M., Rubio-Somoza, I., and Solano, R. 2007. Modulation of plant defenses by ethylene. J. Plant Growth Regul. 26:160-177.

Asami, T., Min, Y. K., Nagata, N., Yamagishi, K., Takatsuto, S., Fujioka, S., Murofushi, N., Yamaguchi, I., and Yoshida, S. 2000. Characterization of brassinazole, a triazole-type brassinosteroid biosynthesis inhibitor. Plant Physiol. 123:93-99.

Azpiroz, R., Wu, Y., LoCascio, J. C., and Feldmann, K. A. 1998. An Arabidopsis brassinosteroid-dependent mutant is blocked in cell elongation. Plant Cell. 10:219-230.

Bajguz, A., and Hayat, S. 2009. Effects of brassinosteroids on the plant responses to environmental stresses. Plant Physiol. Biochem. 47:1-8.

Bancos, S., Nomura, T., Sato, T., Molnar, G., Bishop, G. J., Koncz, C., Yokota, T., Nagy, F., and Szekeres, M. 2002. Regulation of transcript levels of the Arabidopsis cytochrome P450 genes involved in brassinosteroid biosynthesis. Plant Physiol. 130:504-513.

Beckers, G. J. M., and Spoel, S. H. 2006. Fine-tuning plant defense signaling: Salicylate versus jasmonate. Plant Biol. 8:1-10.

Belkhadir, Y., Jaillais, Y., Epple, P., Balsemao-Pires, E., Dangl, J. L., and Chory, J. 2012. Brassinosteroids modulate the efficiency of plant immune responses to microbe-associated molecular patterns. Proc. Natl. Acad. Sci. U.S.A. 109:297-302.

Bouquin, T., Meier, C., Foster, R., Nielsen, M. E., and Mundy, J. 2001. Control of specific gene expression by gibberellin and brassinosteroid. Plant Physiol. 127:450-458.

Bridge, J., Plowright, R. A., and Peng, D. 2005. Nematode parasites of rice. Pages 87-130 in: Plant-Parasitic Nematodes in Subtropical and Tropical Agriculture. M. Luc, R. A. Sikora, and J. Bridge, eds. CAB International, Wallingford, U.K.

Clouse, S. D., and Sasse, J. M. 1998. Brassinosteroids: Essential regulators of plant growth and development. Annu. Rev. Plant Physiol. Plant. Mol. Biol. 49:427-451.

De Vleesschauwer, D., Van Buyten, E., Satoh, K., Balidion, J., Mauleon, R., Choi, I., Vera-Cruz C., Kikuchi, S., and Höfte, M. 2012. Brassinosteroids antagonize gibberellin- and salicylate-mediated root immunity in rice. Plant Physiol. 158:1833-1846.

Divi, U. K., Rahman, T., and Krishna, P. 2010. Brassinosteroid-mediated stress tolerance in Arabidopsis shows interactions with abscisic acid, ethylene and salicylic acid pathways. BMC Plant Biol. 10:151.

Friedrichsen, D. M., Nemhauser, J., Muramitsu, T., Maloof, J. N., Alonso, J., Ecker, J. R., Furuya, M., and Chory, J. 2002. Three redundant brassinosteroid early response genes encode putative bHLH transcription factors required for normal growth. Genetics 162:1445-1456.
Fujioka, S., and Yokota, T. 2003. Biosynthesis and metabolism of brassinosteroids. Annu. Rev. Plant Biol. 54:137-164.

Fujioka, S., Li, J. M., Choi, Y. H., Seto, H., Takatsuto, S., Noguchi, T., Watanabe, T., Kuriyama, H., Yokota, T., Chory, J., and Sakurai, A. 1997. The Arabidopsis deetiolated 2 mutant is blocked early in Brassinosteroid biosynthesis. Plant Cell 9:1951-1962.

Goda, H., Shimada, Y., Asami, T., Fujioka, S., and Yoshida, S. 2002. Microarray analysis of brassinosteroid-regulated genes in Arabidopsis. Plant Physiol. 130:1319-1334.

Grant, M. R., and Jones, J. D. G. 2009. Hormone (dis)harmony moulds plant health and disease. Science 324:750-752.

Hause, B., Stenzel, I., Miersch, O., Maucher, H., Kramell, R., Ziegler, J., and Wasternack, C. 2000. Tissue-specific oxylipin signature of tomato flowers-allene oxide cyclase is highly expressed in distinct flower organs and vascular bundles. Plant J. 24:113-126

He, J. X., Gendron, J. M., Sun, Y., Gampala, S. S. L., Gendron, N., Sun, C. Q., and Wang, Z. Y. 2005. BZR1 is a transcriptional repressor with dual roles in brassinosteroid homeostasis and growth responses. Science 307:1634-1638.

Hong, Z., Ueguchi-Tanaka, M., Shimizu-Sato, S., Inukai, Y., Fujioka, S., Shimada, Y., Takatsuto, S., Agetsuma, M., Yoshida, S., Watanabe, Y., Uozu, S., Kitano, H., Ashikari, M., and Matsuoka, M. 2002. Loss-offunction of a rice brassinosteroid biosynthetic enzyme, C-6 oxidase, prevents the organized arrangement and polar elongation of cells in the leaves and stem. Plant J. 32:495-508.

Hong, Z., Ueguchi-Tanaka, M., Umemura, K., Uozu, S., Fujioka, S., Takatsuto, S., Yoshida, S., Ashikari, M., Kitano, H., and Matsuoka, M. 2003. A rice brassinosteroid-deficient mutant, ebisu dwarf (d2), is caused by a loss of function of a new member of cytochrome P450. Plant Cell 15:2900-2910.

Jung, K.-H., An, G., and Ronald, P. C. 2008. Towards a better bowl of rice: Assigning function to tens of thousands of rice genes. Nat. Rev. Genet. 9:91-101

Khripach, V., Zhabinskii, V., and De Groot, A. 1999. Brassinosteroids: A New Class of Plant Hormones. Academic Press, San Diego, CA, U.S.A.

Khripach, V., Zhabinskii, V., and De Groot, A. 2000. Twenty years of brassinosteroids: Steroidal plant hormones warrant better crops for the XXI century. Ann. Bot. 86:441-447.

Koornneef, A., and Pieterse, C. M. J. 2008. Cross talk in defense signaling. Plant Physiol. 146:839-844.

Kyndt, T., Denil, S., Haegeman, A., Trooskens, G., Bauters, L., Van Criekinge, W., De Meyer, T., and Gheysen, G. 2012a. Transcriptional reprogramming by root knot and migratory nematode infection in rice. New Phytol. 196:887-900.

Kyndt, T., Nahar, K., Haegeman, A., De Vleesschauwer, D., Hofte, M., and Gheysen, G. 2012b. Comparing systemic defense-related gene expression changes upon migratory and sedentary nematode attack in rice. Plant Biol. 14:73-82.

Lee, A., Cho, K., Jang, S., Rakwal, R., Iwahashi, H., Agrawal, G. K., Shim, J., and Han, O. 2004. Inverse correlation between jasmonic acid and salicylic acid during early wound response in rice. Biochem. Biophys. Res. Commun. 318:734-738.

Lee, M. W., Qi, M., and Yang, Y. O. 2001. A novel jasmonic acid-inducible rice $m y b$ gene associates with fungal infection and host cell death. Mol Plant-Microbe Interact. 14:527-535.

Lin, F., Xu, S. L., Ni, W. M., Chu, Z. Q., Xu, Z. H., and Xue, H. W. 2003. Identification of ABA-responsive genes in rice shoots via cDNA macroarray. Cell Res. 13:59-68.

Lorenzo, O., and Solano, R. 2005. Molecular players regulating the jasmonate signaling network. Curr. Opin. Plant Biol. 8:532-540.

Luc, M., Sikora, R. A., and Bridge, J. 2005. Plant Parasitic Nematodes in Subtropical and Tropical Agriculture. CAB International, Wallingford, U.K.

Mandava, N. B. 1988. PLANT GROWTH-PROMOTING BRASSINOSTEROIDS. Annu. Rev. Plant Physiol. Plant. Mol. Biol. 39:23-52.

Mei, C. S., Qi, M., Sheng, G. Y., and Yang, Y. N. 2006. Inducible overexpression of a rice allene oxide synthase gene increases the endogenous jasmonic acid level, PR gene expression, and host resistance to fungal infection. Mol. Plant-Microbe Interact. 19:1127-1137.

Müssig, C., Biesgen, C., Lisso, J., Uwer, U., Weiler, E. W., and Altmann, T. 2000. A novel stress-inducible 12-oxophytodienoate reductase from Arabidopsis thaliana provides a potential link between brassinosteroidaction and jasmonic-acid synthesis. J. Plant Physiol. 157:143-152.

Müssig, C., Fischer, S., and Altmann, T. 2002. Brassinosteroid-regulated gene expression. Plant Physiol. 129:1241-1251.

Nahar, K., Kyndt, T., De Vleesschauwer, D., Hofte, M., and Gheysen, G. 2011. The jasmonate pathway is a key player in systemically induced defense against root knot nematodes in rice. Plant Physiol. 157:305-316

Nakashita, H., Yasuda, M., Nitta, T., Asami, T., Fujioka, S., Arai, Y., Sekimata, K., Takatsuto, S., Yamaguchi, I., and Yoshida, S. 2003. 
Brassinosteroid functions in a broad range of disease resistance in tobacco and rice. Plant J. 33:887-898.

Pfaffl, M. W., Horgan, G. W., and Dempfle, L. 2002. Relative expression software tool (REST (c)) for group-wise comparison and statistical analysis of relative expression results in real-time PCR. Nucleic Acids Res. 30:e36. Published online.

Pieterse, C. M. J., Leon-Reyes, A., Van der Ent, S., and Van Wees, S. C. M. 2009. Networking by small-molecule hormones in plant immunity. Nat. Chem. Biol. 5:308-316.

Rakwal, R., Tamogami, S., Agrawal, G. K., and Iwahashi, H. 2002. Octadecanoid signaling component "burst" in rice (Oryza sativa L.) seedling leaves upon wounding by cut and treatment with fungal elicitor chitosan. Biochem. Biophys. Res. Commun. 295:1041-1045.

Reversat, G., Boyer, J., Sannier, C., and Pando-Bahuon, A. 1999. Use of a mixture of sand and water-absorbent synthetic polymer as substrate for the xenic culturing of plant-parasitic nematodes in the laboratory. Nematology 1:209-212.

Robert-Seilaniantz, A., Grant, M., and Jones, J. D. G. 2011. Hormone crosstalk in plant disease and defense: More than just JASMONATESALICYLATE antagonism. Annu. Rev. Phytopathol. 49:317-343.

Sakurai, A. 1999. Brassinosteroid biosynthesis. Plant Physiol. Biochem. $37: 351-361$

Sakurai, A., and Fujioka, S. 1997. Studies on biosynthesis of brassinosteroids. Biosci. Biotechnol. Biochem. 61:757-762.

Schweizer, P., Buchala, A., Dudler, R., and Metraux, J. P. 1998. Induced systemic resistance in wounded rice plants. Plant J. 14:475-481.

Shan, L., He, P., Li, J., Heese, A., Peck, S. C., Nurnberger, T., Martin, G. B., and Sheen, J. 2008. Bacterial effectors target the common signaling partner BAK1 to disrupt multiple MAMP receptor-signaling complexes and impede plant immunity. Cell Host Microbe 4:17-27.

Shrestha, R., Uzzo, F., Wilson, M. J., and Price, A. H. 2007. Physiological and genetic mapping study of tolerance to root-knot nematode in rice. New Phytol. 176:665-672.

Spoel, S. H., and Dong, X. N. 2008. Making sense of hormone crosstalk during plant immune responses. Cell Host Microbe 3:348-351.

Stelmach, B. A., Muller, A., Hennig, P., Laudert, D., Andert, L., and Weiler, E. W. 1998. Quantitation of the octadecanoid 12-oxo-phy- todienoic acid, a signaling compound in plant mechanotransduction. Phytochemistry 47:539-546.

Stintzi, A., Weber, H., Reymond, P., Browse, J., and Farmer, E. E. 2001. Plant defense in the absence of jasmonic acid: The role of cyclopentenones. Proc. Natl. Acad. Sci. U.S.A. 98:12837-12842.

Symons, G. M., and Reid, J. B. 2004. Brassinosteroids do not undergo long-distance transport in pea. Implications for the regulation of endogenous brassinosteroid levels. Plant Physiol. 135:2196-2206.

Tanabe, S., Ashikari, M., Fujioka, S., Takatsuto, S., Yoshida, S., Yano, M., Yoshimura, A., Kitano, H., Matsuoka, M., Fujisawa, Y., Kato, H., and Iwasaki, Y. 2005. A novel cytochrome P450 is implicated in brassinosteroid biosynthesis via the characterization of a rice dwarf mutant, dwarf11, with reduced seed length. Plant Cell 17:776-790.

van Loon, L. C., Rep, M., and Pieterse, C. M. J. 2006. Significance of inducible defense-related proteins in infected plants. Annu. Rev. Phytopathol. 44:135-162.

Vasyukova, N. J., Chalenko, G. I., Kaneva, I. M., Khripach, V. A., and Ozeretskovskaya, O. L. 1994. Brassinosteroids and potato late blight. Appl. Biochem. Microbiol. 30:464-470. (In Russian).

Whitehead, A. G. 1998. Plant Nematode Control. CAB International, New York.

Williamson, V. M. 1998. Root-knot nematode resistance genes in tomatoes and their potential for future use. Annu. Rev. Phytopathol. 36:277-293.

Williamson, V. M., and Gleason, C. A. 2003. Plant-nematode interactions. Curr. Opin. Plant Biol. 6:327-333.

Yamamuro, C., Ihara, Y., Wu, X., Noguchi, T., Fujioka, S., Takatsuto, S. Ashikari, M., Kitano, H., and Matsuoka, M. 2000. Loss of function of a rice brassinosteroid insensitive 1 homolog prevents internode elongation and bending of the lamina joint. Plant Cell 12:1591-1605.

Yi, H. C., Joo, S., Nam, K. H., Lee, J. S., Kang, B. G., and Kim, W. T. 1999. Auxin and brassinosteroid differentially regulate the expression of three members of the 1-aminocyclopropane-1-carboxylate synthase gene family in mung bean (Vigna radiata L.). Plant. Mol. Biol. 41:443454.

Yokota, T., Nomura, T., and Nakayama, M. 1997. Identification of brassinosteroids that appear to be derived from campesterol and cholesterol in tomato shoots. Plant Cell Physiol. 38:1291-1294. 